\title{
Envelhecimento acelerado em sementes de azevém com e sem solução salina e saturada
}

\author{
Accelerated aging of ryegrass seeds submitted to saturated salt solution
}

\author{
Lilian Madruga de Tunes $^{I^{*}}$ Daniele Cardoso Pedroso $^{I}$ Pablo Gerzson Badinelli ${ }^{I I}$ \\ Lizandro Ciciliano Tavares ${ }^{\text {II }}$ Cassyo de Araújo Rufino ${ }^{\text {II }}$ \\ Antonio Carlos Souza Albuquerque Barros ${ }^{\text {II }}$ \\ Marlove Fátima Brião Muniz
}

RESUMO

O presente trabalho teve por objetivo analisar a metodologia do teste de envelhecimento acelerado para avaliação do potencial fisiológico de sementes de azevém, bem como verificar a possibilidade do uso de solução não saturada e saturada de $\mathrm{NaCl}$ como opção para controle da absorção de água pelas sementes durante a realização do teste, sem reduzir sua sensibilidade. Foram utilizados quatro lotes de sementes, submetidos aos testes de germinação, emergência de plântulas, indice de velocidade de emergência e envelhecimento acelerado, empregando-se os periodos de exposição de 24, 48, 72 e 96h, com e sem solução salina (NaCl). A utilização de solução não saturada e saturada de $\mathrm{NaCl}$ diminui a absorção de água pelas sementes de azevém durante o teste de envelhecimento acelerado, acarretando uma taxa de deterioração menos acentuada e resultados menos drásticos e mais uniformes. A opção $24 \mathrm{~h}$ com solução não saturada (SNS) só conseguiu estratificar os lotes em dois grupos, não devendo ser indicada como promissora. Assim, deve-se usar a opção $48 \mathrm{~h}$ com solução saturada de $\mathrm{NaCl}$ (SSS), que estratificou em três lotes.

Palavras-chave: Lolium multiflorum $\mathrm{L}$., $\mathrm{NaCl}$, germinação, vigor.

\section{ABSTRACT}

The objective of the present study was to evaluate the methodology of the accelerated aging test to evaluate the physiological potential of s ryegrass seeds, as well as verify the possibility of the use of unsaturated and saturated solution of $\mathrm{NaCl}$ as an option for control of water uptake by seeds during the test without reducing its sensitivity. Four lots of ryegrass seeds were tested for germination, seedling emergence, speed emergence and accelerated aging (periods of 24, 48, 72 and 96h, with or without the use of saturated and saline solution of $\mathrm{NaCl}$ ). The use of unsaturated and saturated solution of $\mathrm{NaCl}$ reduces water absorption by ryegrass seeds during the accelerated aging test, resulting in a rate of deterioration is less pronounced, less drastic and more uniform results. Option $24 \mathrm{~h}$ with unsaturated solution (SNS) was only able to stratify plots into two groups and should not be designated as promising. Thus, it should be used the $48 \mathrm{~h}$ saturated $\mathrm{NaCl}$ solution (SSS), which stratified into three lots.

Key words: Lolium multiflorum $\mathrm{L}$., $\mathrm{NaCl}$, germination, vigor.

\section{INTRODUÇÃO}

Nos últimos anos, tem-se evidenciado a necessidade de se obter uma eficiente produção de sementes de espécies forrageiras, como consequência da demanda para incrementar a área de pastagens cultivadas na região Sul do Brasil (TERRA-LOPES et al., 2009). Espécies como azevém têm demonstrado potencial para produção de sementes e um alto valor comercial, merecendo maior atenção em relação à sua qualidade fisiológica.

A qualidade fisiológica das sementes tem sido caracterizada pela germinação e pelo vigor, o qual pode ser definido como a somatória de atributos que conferem à semente o potencial para germinar, emergir e resultar rapidamente em plântulas normais sob ampla diversidade de condições ambientais. Dessa forma, o objetivo básico dos testes de vigor é identificar

'Departamento de Defesa Fitossanitária, Universidade Federal de Santa Maria (UFSM), 97105-900, Santa Maria, RS, Brasil. Email: lilianmtunes@yahoo.com.br.*Autor para correspondência.

"Departamento de Fitotecnia, Universidade Federal de Pelotas (UFPel), Pelotas, RS, Brasil. 
diferenças no potencial fisiológico de lotes de sementes, especialmente daqueles com poder germinativo elevado e semelhante (MARCOS FILHO, 1999b).

O teste de envelhecimento acelerado é um dos mais difundidos para a avaliação do vigor das sementes de várias espécies cultivadas, sendo capaz de proporcionar informações com alto grau de consistência (HAMPTON \& TEKRONY, 1995). Este teste consiste em avaliar a resposta das sementes, por meio do teste de germinação, após estas terem sido submetidas à temperatura elevada e umidade relativa do ar próxima a $100 \%$, por determinado período de exposição (ROSSETO \& MARCOS FILHO, 1995). Baseia-se no fato de que a taxa de deterioração aumenta consideravelmente quando as sementes são expostas a tais condições (HAMPTON \& TEKRONY, 1995). Assim, verifica-se que, em lotes de sementes com baixo vigor, há maior queda na sua viabilidade depois de expostos à situação de estresse pelo envelhecimento (MARCOS FILHO, 1999a), de modo que existe possibilidade de serem estabelecidas diferenças no potencial fisiológico desses lotes (PANOBIANCO \& MARCOS FILHO, 2001).

Um aspecto importante a ser considerado no teste de envelhecimento acelerado é a diferença na absorção de água pelas sementes, pois, quando expostas à atmosfera úmida podem ocorrer variações acentuadas no grau de umidade destas. Pesquisas conduzidas com espécies que possuem sementes de pequeno tamanho têm revelado resultados pouco consistentes devido à variação muito acentuada do grau de umidade das amostras, após o envelhecimento (RAMOS et al., 2004). Nesse sentido, vem sendo estudadas alternativas para a condução do teste de envelhecimento acelerado com sementes dessas espécies, como a substituição da água por soluções de sais. Dependendo da solução utilizada, são obtidos níveis específicos de umidade relativa do ar, permitindo reduzir a taxa de absorção de água, a velocidade e a intensidade de deterioração das sementes (JIANHUA \& McDONALD, 1996), sem reduzir a sensibilidade do teste.

Assim, esta pesquisa teve como objetivo estudar a metodologia do teste de envelhecimento acelerado para avaliação do potencial fisiológico de sementes de azevém, bem como verificar a possibilidade do uso de solução não saturada e saturada de $\mathrm{NaCl}$ como alternativa para controle da absorção de água pelas sementes durante a realização do teste.

\section{MATERIAL E MÉTODOS}

A pesquisa foi conduzida no Laboratório de Análises de Sementes do Departamento de
Fitotecnia, da Universidade Federal de Pelotas, RS. Foram utilizados quatro lotes de sementes de azevém (L1, L2, L3 e L4). Para avaliar o desempenho de cada lote de sementes, conduziram-se os seguintes testes:

Teor de água (TA): conduzido com $3 \mathrm{~g}$ de sementes por subamostra, pelo método da estufa a $105^{\circ} \mathrm{C} \pm 3^{\circ} \mathrm{C}$, durante $24 \mathrm{~h}$ (BRASIL, 2009).

Germinação $(\mathrm{G})$ : conduzido com quatro subamostras de 100 sementes, semeadas em caixas plásticas tipo gerbox, sobre três folhas de papel filtro umedecidas com água destilada e esterilizada, equivalente a 2,5 vezes a massa do papel seco. As caixas foram mantidas em germinador $\left(20^{\circ} \mathrm{C}\right)$. As contagens foram realizadas aos 14 dias após a semeadura, segundo os critérios estabelecidos pelas Regras para Análise de Sementes (BRASIL, 2009). Os resultados foram expressos em porcentagem de plântulas normais.

Emergência de plântulas (EP): foram avaliadas quatro subamostras de 50 sementes para cada lote, semeadas em bandejas de plástico, contendo areia e mantidas a uma temperatura de $20^{\circ} \mathrm{C}$, em ambiente controlado. Foram realizadas irrigações sempre que necessário, e a avaliação ocorreu aos 15 dias após a semeadura, quando a emergência das plântulas tornou-se constante, computando-se a porcentagem de plântulas normais emergidas (NAKAGAWA, 1999).

Índice de velocidade de emergência (IVE): realizado conjuntamente com o teste de emergência de plântulas, através de contagens diárias do número de plântulas emergidas (comprimento de plântula de $1 \mathrm{~cm}$ acima do solo) até o décimo quarto dia. Para cada repetição, foi calculado o índice de velocidade de emergência, somando-se o número de plantas emergidas a cada dia, dividido pelo respectivo número de dias transcorridos a partir da semeadura, conforme MAGUIRE (1962), pela fórmula:

$\mathrm{IVE}=\mathrm{E}_{1}+\mathrm{E}_{2}+\ldots+\mathrm{En}$

$$
\overline{\mathrm{N}}_{1} \overline{\mathrm{N}}_{2} \overline{\mathrm{Nn}}
$$

Sendo: IVE = índice de velocidade de emergência; $E_{1}, E_{2}, E n=$ número de plântulas emergidas, computadas na primeira, na segunda e na última contagem; $\mathrm{N}_{1}, \mathrm{~N}_{2}, \mathrm{Nn}=$ número de dias de semeadura à primeira, segunda e última contagem.

Envelhecimento acelerado tradicional $\left(\mathrm{H}_{2} \mathrm{O}\right)$ : conduzido com a utilização de caixas de plástico transparente (tipo gerbox), contendo $40 \mathrm{ml}$ de água e uma bandeja de tela de alumínio, onde as sementes, após pesagem $(3 \mathrm{~g})$, foram distribuídas formando uma camada uniforme. As caixas foram mantidas em câmara do tipo BOD, a $41^{\circ} \mathrm{C}$, durante $24,48,72$ e $96 \mathrm{~h}$. Decorrido cada período de envelhecimento, quatro subamostras 
de 100 sementes foram submetidas ao teste de germinação, seguindo metodologia descrita anteriormente, com avaliação realizada no quinto dia após a semeadura. Paralelamente foi determinado o teor de água das sementes (descrito anteriormente) após cada período de envelhecimento, para verificar a uniformidade das condições do teste (MARCOS FILHO, 1999b).

Envelhecimento acelerado com uso de solução não saturada de $\mathrm{NaCl}$ (SNS): realizado de forma semelhante ao envelhecimento acelerado tradicional, porém adicionando-se ao fundo das caixas plásticas $40 \mathrm{ml}$ de solução não saturada de sal $(11 \mathrm{~g}$ de $\mathrm{NaCl}$ diluídas em 100ml de água), estabelecendo um ambiente com aproximadamente $94 \%$ de umidade relativa, adaptando a metodologia descrita por JIANHUA \& McDONALD (1996) e determinando conforme a equação de Van't Hoff, descrita por SALISBURY \& ROSS (1992).

Envelhecimento acelerado com o uso de solução saturada de $\mathrm{NaCl}$ (SSS): realizado de forma semelhante ao envelhecimento acelerado tradicional, porém adicionando-se ao fundo das caixas plásticas $40 \mathrm{ml}$ de solução saturada de $\mathrm{NaCl}$ ( $40 \mathrm{~g}$ de $\mathrm{NaCl}$ diluídas em $100 \mathrm{ml}$ de água), estabelecendo ambiente com aproximadamente $76 \%$ de umidade relativa, seguindo a metodologia descrita por JIANHUA \& McDONALD (1996).

Utilizou-se o delineamento experimental inteiramente casualizado. Para comparação das médias, utilizou-se um esquema fatorial $4 \times 4$ (quatro tempos de exposição e quatro lotes de sementes). Para os teores de água, não foram realizadas análises estatísticas. Os resultados foram submetidos à análise de variância, e as médias comparadas pelo teste de Tukey $(a=0,05)$, empregando-se o programa de análises estatísticas Sisvar (Ferreira, 2000).

\section{RESULTADOS E DISCUSSÃO}

Os dados referentes ao teor de água das sementes de azevém foram semelhantes para os quatro lotes, ou seja, os valores ficaram entre 12,03 e 12,18\% de umidade (Tabela 1). Este fato é importante para a execução das avaliações de envelhecimento acelerado, considerando-se que a uniformização do teor de água das sementes é imprescindível para a padronização das avaliações e obtenção de resultados consistentes (MARCOS FILHO, 2005), pois, dentro de certos limites, as sementes mais úmidas são mais afetadas pelas condições do envelhecimento acelerado. De acordo com PEDROSO (2009), quando o teor de água das sementes é relativamente baixo, como ocorreu nos lotes
Tabela 1 - Qualidade inicial dos lotes das sementes de azevém pelo teor de água (TA), teste de germinação (G), emergência de plântulas (EP) e índice de velocidade de emergência (IVE).

\begin{tabular}{|c|c|c|c|c|}
\hline & $\mathrm{TA}$ & G & EP & IVE \\
\hline 1 & 12,15 & $90 \mathrm{a}$ & $83 \mathrm{a}$ & $12,7 \mathrm{a}$ \\
\hline 2 & 12,08 & $82 \mathrm{~b}$ & $77 \mathrm{a}$ & $12,7 \mathrm{a}$ \\
\hline 3 & 12,18 & $65 \mathrm{c}$ & $60 \mathrm{~b}$ & $12,1 \mathrm{a}$ \\
\hline 4 & 12,03 & $57 \mathrm{c}$ & $49 \mathrm{c}$ & $7,56 \mathrm{~b}$ \\
\hline CV (\%) & & 9,08 & 8,17 & 4,57 \\
\hline
\end{tabular}

"Médias seguidas de mesma letra na coluna não diferem entre si pelo teste de Tukey a $5 \%$ de probabilidade.

das sementes de azevém, é permiteida uma maior confiabilidade aos resultados obtidos nos testes de qualidade fisiológica.

Os resultados do teste de germinação (Tabela 1) indicaram diferenças entre os lotes de sementes de azevém, destacando-se o lote 1 como de qualidade superior; os lotes 3 e 4 de qualidade inferior, e o lote 2 como de qualidade intermediária. O teste de emergência de plântulas foi o que diferenciou todos os lotes, evidenciando os lotes 1 e 2 como de potencial fisiológico superior, seguidos pelos lotes 3 e 4 em ordem decrescente quanto à qualidade. $\mathrm{O}$ teste de índice de velocidade de emergência apenas diferenciou o lote 4, sendo este de inferior qualidade quando comparado aos demais. A discordância entre os resultados obtidos nos testes sugere, justamente, a necessidade de realização do maior número possível de testes antes de classificar os lotes quanto ao potencial fisiológico, pois cada teste tem um princípio diferente e fornece informações complementares para a decisão a respeito do destino final de cada lote de semente.

Examinando-se os resultados do teste de envelhecimento acelerado (Tabela 2), tanto o procedimento tradicional $(48 \mathrm{~h})$, com solução não saturada de $\mathrm{NaCl}$ (72h) quanto com solução saturada de $\mathrm{NaCl}$ (48 e 96h), observa-se que estes permitiram a estratificação dos lotes de sementes de azevém quanto ao vigor, proporcionando a mesma separação dos lotes verificada pela emergência de plântulas (Tabela 1) (testes de vigor diferentes com a mesma classificação).

Os resultados obtidos no teste de envelhecimento acelerado conduzido de forma tradicional (72h), com solução salina não saturada (48h) e com solução saturada de $\mathrm{NaCl}$ (24h) separaram os lotes em maior número de níveis de vigor, pois, além de indicar o lote 1 como de potencial fisiológico superior, também detectaram diferenças, entre os lotes 2, 3 e 4, 
Tabela 2 - Porcentagem de germinação das sementes de azevém após o teste de envelhecimento acelerado tradicional $\left(\mathrm{H}_{2} \mathrm{O}\right)$, solução não saturada (SNS) e solução saturada de $\mathrm{NaCl}$ (SSS), durante o período de exposição de 24, 48, 72 e 96h.

\begin{tabular}{|c|c|c|c|c|c|c|c|c|c|c|c|c|}
\hline \multirow{2}{*}{ Lotes } & \multicolumn{4}{|c|}{----------------Tradicional---------------- } & \multicolumn{4}{|c|}{-----------------SNS----------------- } & \multicolumn{4}{|c|}{------------------SSS------------------ } \\
\hline & $24 \mathrm{~h}$ & $48 \mathrm{~h}$ & $72 \mathrm{~h}$ & $96 \mathrm{~h}$ & $24 \mathrm{~h}$ & $48 \mathrm{~h}$ & $72 \mathrm{~h}$ & $96 \mathrm{~h}$ & $24 \mathrm{~h}$ & $48 \mathrm{~h}$ & $72 \mathrm{~h}$ & $96 \mathrm{~h}$ \\
\hline & \multicolumn{12}{|c|}{$\%$} \\
\hline 1 & $79 \mathrm{a}$ & $64 \mathrm{a}$ & $41 \mathrm{a}$ & $1 \mathrm{a}$ & $83 \mathrm{a}$ & $85 \mathrm{a}$ & $78 \mathrm{a}$ & $61 \mathrm{a}$ & $88 \mathrm{a}$ & $84 \mathrm{a}$ & $80 \mathrm{a}$ & $72 \mathrm{a}$ \\
\hline 2 & $66 \mathrm{~b}$ & $59 \mathrm{a}$ & $29 \mathrm{~b}$ & $0 \mathrm{a}$ & $80 \mathrm{a}$ & $78 \mathrm{~b}$ & $70 \mathrm{a}$ & $57 \mathrm{a}$ & $80 \mathrm{~b}$ & $80 \mathrm{a}$ & $78 \mathrm{a}$ & $70 \mathrm{a}$ \\
\hline 3 & $58 \mathrm{~b}$ & $45 \mathrm{~b}$ & $17 \mathrm{c}$ & $0 \mathrm{a}$ & $55 \mathrm{~b}$ & $60 \mathrm{c}$ & $52 \mathrm{~b}$ & $55 \mathrm{a}$ & $63 \mathrm{c}$ & $60 \mathrm{~b}$ & $58 \mathrm{~b}$ & $57 \mathrm{~b}$ \\
\hline 4 & $44 \mathrm{c}$ & $34 \mathrm{c}$ & $5 \mathrm{~d}$ & $0 \mathrm{a}$ & $50 \mathrm{~b}$ & $55 \mathrm{~d}$ & $42 \mathrm{c}$ & $40 \mathrm{~b}$ & $57 \mathrm{~d}$ & $53 \mathrm{c}$ & $53 \mathrm{~b}$ & $42 \mathrm{c}$ \\
\hline CV (\%) & \multicolumn{4}{|c|}{11,02} & \multicolumn{4}{|c|}{8,08} & \multicolumn{4}{|c|}{10,15} \\
\hline
\end{tabular}

${ }^{*}$ Médias seguidas de mesma letra na coluna não diferem entre si pelo teste de Tukey a $5 \%$ de probabilidade.

não verificadas nos testes de emergência de plântulas e de índice de velocidade de emergência. A adição do $\mathrm{NaCl}$ faz com que as sementes não absorvam muita água (proporciona uma menor umidade relativa do ar quando comparado apenas com água) e sofram um processo de deterioração acentuado, mesmo diante de uma metodologia que causa um estresse nas sementes com a utilização de alta temperatura.

No entanto, ao se analisar os valores de germinação após o envelhecimento tradicional, notouse elevada redução na porcentagem de plântulas normais, indicando que o procedimento que utiliza $72 \mathrm{~h}$ de exposição não foi o mais adequado para sementes de azevém. Resultados semelhantes foram obtidos por RAMOS et al. (2004) e TUNES et al. (2009) em sementes de rúcula e cevada, respectivamente, quando constataram que o estresse provocado pelo teste de envelhecimento acelerado tradicional por um período de $72 \mathrm{~h}$ ocasionou uma redução expressiva da germinação destas sementes.

O mais adequado seria utilizar o período de 48h de exposição em solução não saturada de $\mathrm{NaCl}$ ou $24 \mathrm{~h}$ em solução saturada de $\mathrm{NaCl}$, pois o menor período de execução é uma característica desejável em um teste de vigor, pois possibilita a economia de energia elétrica pelo equipamento, além de fornecer resultados em menor período de tempo.
Os resultados médios do teor de água atingido após a realização do teste de envelhecimento acelerado tradicional, solução não saturada e saturada de $\mathrm{NaCl}$ estão na tabela 3. Observa-se que as sementes de azevém envelhecidas no procedimento tradicional atingiram teores de água mais elevados e com maiores variações, diferindo valores de até 6,9 pontos percentuais (p.p.), que excedem os limites toleráveis de 3 a 4p.p., indicados por MARCOS FILHO (1999b). No procedimento tradicional, as sementes absorveram maior percentual de água quando comparadas àquelas da metodologia com uso de solução não saturada e saturada de $\mathrm{NaCl}$. Da mesma forma, RODO et al. (2000) verificaram, para sementes de cenoura, variações de 5,0 a 9,2p.p., consideradas excessivas, ao final do envelhecimento acelerado tradicional.

Por outro lado, verificou-se que o uso de solução salina não saturada reduziu a velocidade de absorção de água pelas sementes durante o período de envelhecimento, não excedendo a variação de 2,2p.p. do teor de água entre os lotes envelhecidos. O mesmo ocorreu com o envelhecimento com uso de solução saturada de $\mathrm{NaCl}$, com variação máxima de 1,2p.p. As condições de envelhecimento acelerado com solução não saturada e saturada de $\mathrm{NaCl}$ promoveram efeitos menos drásticos, pois, ao atingir menores teores de água (máximo de 19,36\%(SNS)e 14,08\%(SSS), enquanto

Tabela 3 - Teor de água (TA) das sementes de azevém após o teste de envelhecimento acelerado tradicional $\left(\mathrm{H}_{2} \mathrm{O}\right)$, solução não saturada (SNS) e solução saturada de $\mathrm{NaCl}$ (SSS), durante o período de exposição de 24, 48, 72 e 96h.

\begin{tabular}{|c|c|c|c|c|c|c|c|c|c|c|c|c|}
\hline \multirow{2}{*}{ Lotes } & \multicolumn{4}{|c|}{------------------Tradicional------------------ } & \multicolumn{4}{|c|}{--------------------SNS-------------------- } & \multicolumn{4}{|c|}{--------------------SSS-------------------' } \\
\hline & $24 \mathrm{~h}$ & $48 \mathrm{~h}$ & $72 \mathrm{~h}$ & $96 \mathrm{~h}$ & $24 \mathrm{~h}$ & $48 \mathrm{~h}$ & $72 \mathrm{~h}$ & $96 \mathrm{~h}$ & $24 \mathrm{~h}$ & $48 \mathrm{~h}$ & $72 \mathrm{~h}$ & $96 \mathrm{~h}$ \\
\hline & \multicolumn{12}{|c|}{$\%$} \\
\hline 1 & 20,34 & 23,72 & 34,01 & 38,11 & 14,21 & 16,62 & 17,15 & 19,36 & 12,27 & 12,33 & 13,19 & 13,01 \\
\hline 2 & 21,13 & 23,30 & 35,42 & 40,12 & 14,35 & 16,11 & 17,00 & 18,55 & 12,45 & 12,47 & 12,86 & 13,14 \\
\hline 3 & 22,15 & 25,03 & 37,09 & 43,60 & 16,08 & 18,22 & 18,82 & 17,23 & 13,03 & 13,15 & 13,79 & 14,00 \\
\hline 4 & 22,26 & 27,19 & 38,36 & 45,03 & 15,44 & 18,27 & 18,87 & 17,75 & 13,21 & 13,27 & 14,03 & 14,08 \\
\hline
\end{tabular}

Ciência Rural, v.41, n.1, jan, 2011. 
o tradicional chegou a $45,03 \%$ ), a deterioração das sementes foi atenuada em relação ao método tradicional. Isso também foi verificado por TORRES \& MARCOS FILHO (2003), trabalhando com sementes de melão.

Resultados semelhantes foram encontrados por TORRES (2004), com a utilização de solução saturada de $\mathrm{NaCl}$ no teste de envelhecimento acelerado com sementes de erva doce, em que a redução da velocidade de absorção de água pelas sementes acarretou deterioração menos acentuada e resultados menos drásticos e mais uniformes que os obtidos com o procedimento tradicional. Este método também foi eficiente para avaliação do vigor de sementes de melancia (BHERING et al., 2003), tomate (PANOBIANCO \& MARCOS FILHO, 2001), rúcula (RAMOS et al., 2004) e cevada (TUNES et al., 2009).

Os resultados deste trabalho confirmam que o uso de solução salina saturada ou não saturada de $\mathrm{NaCl}$ contribui para o aprimoramento da metodologia do teste de envelhecimento acelerado na avaliação do vigor de sementes de azevém, pois, além de utilizar o mesmo equipamento do procedimento tradicional, proporciona condições para a diminuição da absorção de água pelas sementes e de maneira mais uniforme.

\section{CONCLUSÃO}

A utilização de solução não saturada e saturada de $\mathrm{NaCl}$ diminui a absorção de água e a taxa de deterioração das sementes de azevém durante o teste de envelhecimento acelerado. A opção $48 \mathrm{~h}$ com solução saturada de $\mathrm{NaCl}$ (SSS) é a que deve ser utilizada porque promove uma melhor estratificação dos lotes de sementes de azevém.

\section{REFERÊNCIAS}

BHERING, M.C. et al. Avaliação do vigor de sementes de melancia (Citrullus lanatus Scherad.) pelo teste de envelhecimento acelerado. Revista Brasileira de Sementes, v.25, n.1, p.1-6, 2003. Disponível em: <http://www.scielo.br/ pdf/rbs/v28n3/10.pdf>. Acesso em: 27 jul. 2010. doi: 10.1590/ S0101-31222006000300010.

BRASIL. Ministério da Agricultura, Pecuária e Abastecimento. Regras para análise de sementes. Brasília, 2009. 399p.

FERREIRA, D.F. Análises estatísticas por meio do SISVAR para Windows versão 4.0. In: REUNIÃO ANUAL DA REGIÃO BRASILEIRA DA SOCIEDADE INTERNACIONAL DE BIOMETRIA, 45., São Carlos, SP. Anais... São Carlos: UFSCAR, 2000. p.225-258.

HAMPTON, J.G.; TEKRONY, D.M. Handbook of vigour test methods. 3.ed. Zurich: ISTA, 1995. 117p.

JIANHUA, Z.; MCDONALD, M.B. The saturated salt accelerated aging test for small-seeded crops. Seed Science and Technology, v.25, n.1, p.123-131, 1996.
MAGUIRE, J.D. Speed of germination-aid in selection and evaluation for seedling emergence and vigour. Crop Science, v.2, n.1, p.176-177, 1962 .

MARCOS FILHO, J. Teste de envelhecimento acelerado. In: KRZYZANOWSKI, F.C. et al. Vigor de sementes. Conceitos e testes. Londrina: ABRATES, 1999a. p.1.1-1.21.

MARCOS FILHO, J. Testes de vigor: importância e utilização. In: KRZYZANOWSKI, F.C. et al. Vigor de sementes. Conceitos e testes. Londrina: ABRATES, 1999b. p.3.1-3.24.

MARCOS FILHO, J. Fisiologia de sementes de plantas cultivadas. Piracicaba: FEALQ, 2005. 495p.

NAKAGAWA, J. Testes de vigor baseado do desempenho das plântulas. In: KRZYZANOWSKI, F.C. et al . Vigor de sementes. Conceitos e testes. Londrina: ABRATES, 1999. p. 2-1/2-24.

PANOBIANCO, M.; MARCOS FILHO, J. Envelhecimento acelerado e deterioração controlada em sementes de tomate. Scientia Agricola, v.58, n.3, p.525-531, 2001. Disponível em: <http://www.scielo.br/pdf/sa/v58n3/a14v58n3.pdf>. Acesso em: 11 abr. 2010. doi: 10.1590/S0103-90162001000300014.

PEDROSO, D.C. Associação de Alternaria spp. com sementes de apiáceas: métodos de inoculação e influência na qualidade fisiológica. 2009. 118f. Dissertação (Mestrado em Agronomia) - Universidade Federal de Santa Maria, Santa Maria, RS

RAMOS, N.P. et al. Envelhecimento acelerado em sementes de rúcula (Eruca sativa L.). Revista Brasileira de Sementes, v.26, n.1, p.98-103, 2004. Disponível em: <http://www.scielo.br/ pdf/rbs/v26n1/a15v26n1.pdf>. Acesso em: 20 jul. 2010. doi: 10.1590/S0101-31222004000100015.

RODO, A.B. et al. Metodologia alternativa do teste de envelhecimento acelerado para sementes de cenoura. Scientia Agricola, v.57, n.1, p.289-292, 2000.

ROSSETTO, C.A.V.; MARCOS FILHO, J. Comparação entre os métodos de envelhecimento acelerado e de deterioração controlada para avaliação da qualidade fisiológica de sementes de soja. Scientia Agricola, v.52, n.2, p.123-131, 1995.

SALISBURY, F.B.; ROSS, C.W. Plant physiology. 4.ed. Belmont: Wadsworth, 1992. 682p.

TERRA-LOPES, M.L. et al. Sistema de integração lavourapecuária: efeito do manejo da altura em pastagem de aveia preta e azevém anual sobre o rendimento da cultura da soja. Ciência Rural, v.39, n.5, p.1499-1506, 2009. Disponível em: <http:// www.scielo.br/scielo.php? script=sci_abstract\&pid=S010384 $782009000500030 \& \operatorname{lng}=\mathrm{pt} \& \mathrm{nrm}=\mathrm{iso} \& \mathrm{t} \operatorname{lng}=\mathrm{pt}>$. Acesso em: 20 jul. 2010. doi: 10.1590/S0103-84782009005000096.

TORRES, S.B. Teste de envelhecimento acelerado em sementes de erva-doce. Revista Brasileira de Sementes, v.26, n.2, p.20-24, 2004. Disponível em: <http://www.scielo.br/pdf/rbs/ v26n2/24485.pdf>. Acesso em: 20 jul. 2010. doi: 10.1590/ S0101-31222004000200004.

TORRES, S.B.; MARCOS FILHO, J. Accelerated aging of melon seeds. Scientia Agricola, v.60, n.1, p.77-82, 2003. Disponível em: <http://www.scielo.br/pdf/sa/v60n1/14546.pdf >. Acesso em: 20 jul. 2010. doi: 10.1590/S0103-90162003000100012.

TUNES, L.M. et al. Teste de envelhecimento acelerado em cevada. Magistra, v.21, n.2, p.111-119, 2009. 